\title{
Identification of Orthodontic Extraction Predictors in End-On Class II Malocclusion
}

\author{
Batool Ali ${ }^{1}$ \\ Waqar Jeelani \\ Attiya Shaikh ${ }^{3}$ \\ Tabassum Ahsan Quadeer ${ }^{4}$ \\ Javeria Ali Khan ${ }^{5}$
}

\author{
BDS, FCPS \\ BDS, FCPS \\ BDS, M.Orth, FCPS \\ BDS, FCPS \\ BDS, FCPS
}

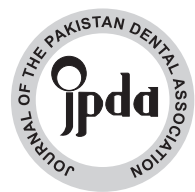

OBJECTIVE: This study was designed to determine the different treatment predictors which help in the extraction and non-extraction decision of an end-on Class II malocclusion case.

METHODOLOGY: The pretreatment records of 240 adult subjects aged 15-40 years with bilateral end-on Class II molar relationship were retrospectively selected and categorized under extraction (120) and non-extraction (120) treatment categories. The extraction cases were planned for different combinations of premolar extractions. The independent variables i.e., the cephalometric and orthodontic cast measurements were obtained from the recruited sample. Binary logistic regression analysis was applied using SPSS software.

RESULTS: Increased upper and lower incisor inclinations ( $p<0.001)$ and procumbent upper lip $(p=0.004)$ was statistically significant in the extraction group. According to the regression model, the odds of extraction treatment were 1.12 times greater than non-extraction treatment for every one degree increase in upper and lower incisor inclinations, respectively. The chances of extraction treatment were 1.6 times higher than non-extraction treatment for every $1 \mathrm{~mm}$ increase in the distance of upper lip to S-plane.

CONCLUSIONS: The upper and lower incisors inclinations and upper lip position are the critical factors affecting the extraction decision in adult patients with end-on Class II molar relationships. Overjet, dental crowding and the vertical growth pattern were found to be clinically insignificant in opting for an extraction treatment plan for such cases.

KEYWORDS:Treatment, Angle Class II, tooth extraction, non-extraction

HOW TO CITE: Ali B, Jeelani W, Shaikh A, Quadeer TA, Khan JA. Identification of orthodontic extraction predictors in endon class II malocclusion. J Pak Dent Assoc 2021;30(3):178-182 .

DOI: https://doi.org/10.25301/JPDA.303.178

Received: 13 March 2021, Accepted: 21 June 2021

\section{INTRODUCTION}

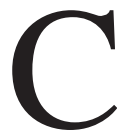
lass II malocclusion can be treated via different therapeutic approaches depending on the severity of malocclusion and growth status of an individual. Patients presenting in the prepubertal or pubertal growth period are routinely treated with dento-facial orthopedics

1. Assistant Professor, Department of Orthodontics, Dow Dental College, Dow University of Health Sciences, Karachi, Pakistan.

2. Assistant Professor, Department of Orthodontics, Bakhtawar Amin Dental College and Hospital, Northern Bypass Multan, Pakistan.

3. Professor, Department of Orthodontics, The Liaquat College of Medicine and Dentistry,Karachi, Pakistan.

4. Associate Professor, Department of Orthodontics, Bahria University Medical and Dental College Karachi, Pakistan.

5. Assistant Professor, Department of Operative Dentistry, Dow Dental College, Dow University of Health Sciences, Karachi, Pakistan

Corresponding author: “Dr. Batool Ali” < batool.ali@duhs.edu.pk > to correct the skeletal deformity. ${ }^{1,2}$ Adult patients with no growth potential require comprehensive treatment planning to decide between orthodontic camouflage and surgical treatment. Non extraction decision with molar distalization, extraction patterns including four premolars, one from each quadrant, or in special scenarios, asymmetric extraction or molar extractions are carried out to achieve acceptable dento-facial esthetics with stable occlusion. ${ }^{3,4}$

The orthodontic diagnosis and treatment planning involves the process of identifying the correct etiology and designing an individualized treatment plan. The decision to extract or not extract is greatly influenced by parameters like incisor proclination, lip incompetency, procumbent or recumbent lip profile, level of crowding in one or both arches, midline discrepancy, molar relationship and the complexity of the malocclusion. ${ }^{5-8}$ Although the above mentioned factors play an essential role in planning process, 
yet, a comprehensive plan must be generated for every individual without limiting it to the obvious quantitative variables.

The rationale behind conducting this study was that class II correction protocols till date remain a controversial issue and orthodontists agree that stability of a class II corrected case is as important as the facial harmony and esthetic balance. Hence, the variables that could help in planning a case with the best possible patient-oriented approach that not only improvises the orofacial esthetics but also gives long term stability to a class II malocclusion needs yet to be identified. According to a pertinent literature survey, none of the studies have yet uncovered the variables which account for favoring an extraction approach over a non-extraction approach and vice versa in end on Class II cases that could accelerate the decision making process and establish evidence based predictors for planning such cases. Due to the absence of a proper guideline or potent evidence, the treatment planning process generally is more dependent on a subjective approach via clinician's personal experiences, treatment philosophy and mode of training. ${ }^{9-12}$

Therefore, the objective of this study was designed to review the pretreatment records of patients with end-on Class II malocclusion and compare the initial morphologic characteristics of patients diagnosed and planned by two expert clinicians in order to determine the different treatment predictors which can help a clinician in the extraction and non-extraction decision of an end-on Class II malocclusion case.

\section{METHODOLOGY}

Records of 1500 orthodontic patients were screened from the orthodontic clinics of a total of 400 pre-treatment records having Class II malocclusion with a half unit molar relationship was sorted. A molar relationship was considered end-on if the mesio-buccal cusp tip of maxillary first molar in occlusion was $\geq 3 \mathrm{~mm}$ and $\leq 4 \mathrm{~mm}$ mesial to the buccal groove of lower first molar. ${ }^{1}$ An ethical exemption was taken from the institutional review board before data collection (4078-Sur-ERC-17). The sample size was calculated as 120 records in each group using Open Epi software using the mean values of Li:NB distance from a study conducted by Bishara et $\mathrm{al}^{13}$ with a value of $0.6 \pm 1.8 \mathrm{~mm}$ for the extraction group and $1.2 \pm 1.5 \mathrm{~mm}$ for the non-extraction group at a CI of $95 \%$ and keeping the power of study as $80 \%$. The orthodontic records were carefully selected using simple random sampling method. Orthodontic record of each patient was critically evaluated on a routine basis by a team of orthodontists to formulate the most efficient treatment plan for that particular individual. The extraction cases selected for the study were those planned by expert clinicians for different combinations of premolar extractions. The non-extraction cases planned to be treated by molar distalization were selected for the study.

The following criteria was established for the orthodontic records to be included in the current study. Good quality complete dental records with healthy dentition having bilateral end-on Class II molar relations. Subjects with any history of trauma, syndromes or craniofacial anomalies, and those undergone previous orthodontic treatment were excluded from the study. The lateral cephalograms carried out for orthodontic analysis were recorded in natural head position by the same machine for each individual. The head was positioned parallel to the Frankfurt horizontal plane with teeth positioned in centric occlusion. These radiographs were standardized with rigid head fixation, $152-\mathrm{cm}$

source to the mid-sagittal plane distance and $15 \mathrm{~cm}$ film to the mid-sagittal plane distance using Orthoralix ${ }^{\circledR} 9200$ (Gendex-KaVo, Milan, Italy). ${ }^{14}$

The mill metric measurements (overbite, overjet, dental midlines, maxillary and mandibular space analysis) on dental casts were carried out using a digital vernier caliper (0-150mm ME00183, Dentaurum, Pforzheim, Germany) with accuracy of $0.02 \mathrm{~mm}$ and reliability of $0.01 \mathrm{~mm}$ as per manufacturer's specification. The measurement done on orthodontic casts included: overjet, overbite, upper and lower dental midlines and maxillary (Mx-TSALD) and mandibular tooth size arch length discrepancy (Md-TSALD). Lateral cephalograms were traced, landmarks were identified and planes were constructed according to the standard definitions given in orthodontic books and literature. Cephalometric measurements derived from the landmarks and planes included: SNA, SNB, ANB, Wits appraisal, Nasion perpendicular to point A, Nasion perpendicular to Pogonion SN-Go.Gn, FHMP, lower anterior facial height (LAFH), UI-SN, IMPA, nasolabial angle, and relationship of upper and lower lips to $\mathrm{E}$ and $\mathrm{S}$ planes.

Repeated measurements of 25 randomly selected dental casts and cephalometric radiographs were made 2 months after the initial assessment and ICC was used to evaluate the reliability of repeated measurements. The ICC showed a value of 0.80 for the variables assessed.

\section{STATISTICAL ANALYSIS}

The statistical analysis was undertaken using the SPSS software for Windows (version 22 .0; SPSS, Chicago, Ill). Means and SD's were calculated for the independent variables. Binary logistic regression was applied to evaluate 
the effect of independent variables on the treatment outcome. The binary logistic regression technique is most often used to model the relationship between a binary outcome variable and a set of covariates. The binary outcome variables was the treatment decision, whereas, the measurements attained from cephalometric analysis and orthodontic casts were categorized as independent variables. Multicollinearity of the independent variables was also assessed. A value of greater than 0.83 was considered as highly significantly correlated, hence, only one of the variable was used for the final logistic model.

\section{RESULTS}

The descriptive statistics are summarized in Table 1. The ANB angle, Nasion perpendicular to Pogonion, the vertical facial pattern, the upper and lower incisor

Table 1: Descriptive statistics for the study groups.

\begin{tabular}{|c|c|c|c|}
\hline \multicolumn{2}{|r|}{ Variables } & \multirow{2}{*}{$\begin{array}{c}\begin{array}{c}\text { Non-Extraction } \\
\text { Group } \\
(\mathrm{n}=\mathbf{1 2 0}, \text { Means } \pm \text { SD })\end{array} \\
80.2 \pm 7.79\end{array}$} & \multirow{2}{*}{$\begin{array}{c}\begin{array}{c}\text { Extraction Group } \\
(\mathrm{n}=\mathbf{1 2 0}, \text { Means } \pm \text { SD })\end{array} \\
\mathbf{8 2 . 4} \pm \mathbf{4 . 1 3}\end{array}$} \\
\hline \multirow{9}{*}{ Skeletal } & SNA (degrees) & & \\
\hline & SNB (degrees) & $75.7 \pm 3.70$ & $76.0 \pm 3.77$ \\
\hline & ANB (degrees) & $5.02 \pm 2.05$ & $6.27 \pm 3.21$ \\
\hline & Wits (mm) & $2.15 \pm 3.19$ & $2.92 \pm 4.42$ \\
\hline & Nasion perpendicular to point $\mathbf{A}(\mathrm{mm})$ & $-0.40 \pm 3.15$ & $0.03 \pm 3.75$ \\
\hline & Nasion perpendicular to Pogonion $(\mathrm{mm})$ & $-8.04 \pm 6.33$ & $-9.87 \pm 7.25$ \\
\hline & SN to Go-Gn (degrees) & $30.8 \pm 6.09$ & $30.9 \pm 6.11$ \\
\hline & FMA (degrees) & $24.4 \pm 6.36$ & $25.8 \pm 5.95$ \\
\hline & LAFH / TAFH ratio (\%) & $55.47 \pm 3.24$ & $56.27 \pm 3.18$ \\
\hline \multirow{8}{*}{ Dental } & UI-SN (degrees) & $106.3 \pm 8.85$ & $112.4 \pm 7.83$ \\
\hline & IMPA (degrees) & $97.8 \pm 7.78$ & $102.6 \pm 6.94$ \\
\hline & Overjet $(\mathbf{m m})$ & $6.59 \pm 2.94$ & $8.35 \pm 3.18$ \\
\hline & Overbite (mm) & $4.50 \pm 1.79$ & $4.13 \pm 2.71$ \\
\hline & UDM discrepancy (mm) & $-0.05 \pm 0.61$ & $0.00 \pm 0.92$ \\
\hline & LDM discrepancy (mm) & $-0.05 \pm 1.13$ & $-0.06 \pm 1.35$ \\
\hline & Maxillary TSALD (mm) & $-1.42 \pm 5.52$ & $-2.48 \pm 5.32$ \\
\hline & Mandibular TSALD (mm) & $-2.17 \pm 4.20$ & $-3.88 \pm 4.69$ \\
\hline \multirow{5}{*}{ Soft Tissue } & E-plane to Upper lip (mm) & $-2.61 \pm 2.98$ & $-0.77 \pm 3.10$ \\
\hline & E-plane to Lower lip (mm) & $-1.10 \pm 3.08$ & $-0.06 \pm 3.40$ \\
\hline & S-plane to Upper lip (mm) & $0.66 \pm 2.37$ & $2.39 \pm 2.51$ \\
\hline & S-plane to Lower lip (mm) & $1.01 \pm 2.72$ & $2.95 \pm 3.08$ \\
\hline & Nasolabial Angle (degrees) & $101.5 \pm 12.25$ & $107.5 \pm 7.83$ \\
\hline
\end{tabular}

$\mathrm{UDM}=$ upper dental midline, $\mathrm{LDM}=$ lower dental midline

inclinations, overjet, Md-TSALD and the relation of both the lips to E-plane and S-plane, respectively, were significantly different between both the non-extraction and extraction groups in the univariate model generated after the initial regression analysis as shown in Table 2 . Table 3 shows the final multivariable model. According to the statistical results, one skeletal, one soft tissue and three
Table 2: Univariate regression model of skeletal, dental and soft tissue variables

\begin{tabular}{|c|c|c|c|c|}
\hline \multicolumn{2}{|r|}{ Variables } & $\begin{array}{l}\text { Odds Ratio } \\
\text { (OR) }\end{array}$ & P - Value & $\begin{array}{l}\text { Confidence } \\
\text { Interval (CI) }\end{array}$ \\
\hline \multirow{9}{*}{ Skeletal } & SNA (degrees) & 1.10 & 0.003 & $1.03-1.18$ \\
\hline & SNB (degrees) & 1.02 & 0.46 & $0.95-1.09$ \\
\hline & ANB (degrees) & 1.29 & $<0.001^{* *}$ & $1.14-1.47$ \\
\hline & Wits (mm) & 1.05 & 0.127 & $0.98-1.12$ \\
\hline & Nasion perpendicular to point $\mathbf{A}(\mathrm{mm})$ & 1.03 & 0.32 & $0.96-1.11$ \\
\hline & Nasion perpendicular to Pogonion (mm) & 0.96 & $0.04 *$ & $0.92-0.99$ \\
\hline & SN to Go-Gn (degrees) & 1.00 & 0.86 & $0.96-1.04$ \\
\hline & FMA (degrees) & 1.03 & 0.08 & $0.99-1.08$ \\
\hline & LAFH /TAFH ratio (\%) & 1.08 & $0.05^{*}$ & $0.99-1.17$ \\
\hline \multirow{8}{*}{ Dental } & UI-SN (degrees) & 1.09 & $<0.001^{* *}$ & $1.05-1.13$ \\
\hline & IMPA (degrees) & 1.09 & $<0.001^{* *}$ & $1.05-1.13$ \\
\hline & Overjet (mm) & 1.20 & $<0.001^{\text {*ᄎ }}$ & $1.10-1.32$ \\
\hline & Overbite (mm) & 0.93 & 0.21 & $0.83-1.04$ \\
\hline & UDM discrepancy (mm) & 1.10 & 0.56 & $0.79-1.52$ \\
\hline & LDM discrepancy (mm) & 1.07 & 0.47 & $0.87-1.32$ \\
\hline & Maxillary TSALD (mm) & 0.96 & 0.13 & $0.91-1.01$ \\
\hline & Mandibular TSALD (mm) & 0.91 & $0.004^{*}$ & $0.86-0.97$ \\
\hline \multirow{5}{*}{ Soft Tissue } & E-plane to Upper lip (mm) & 1.21 & $<0.001 * *$ & $1.11-1.33$ \\
\hline & E-plane to Lower lip (mm) & 1.21 & $<0.001^{* *}$ & $1.12-1.32$ \\
\hline & S-plane to Upper lip (mm) & 1.34 & $<0.001^{* *}$ & $1.19-1.50$ \\
\hline & S-plane to Lower lip (mm) & 1.26 & $<0.001^{\text {** }}$ & $1.14-1.38$ \\
\hline & Nasolabial Angle (degrees) & 1.00 & 0.483 & $0.99-1.01$ \\
\hline
\end{tabular}

$\mathrm{N}=240$, Logistic regression analysis, *p-value $\leq 0.05$, **p-value $<0.001 \mathrm{UDM}=$ upper dental midline, $\mathrm{LDM}=$ lower dental midline

Table 3: Multivariable Model

\begin{tabular}{|c|l|c|c|c|c|}
\hline \multicolumn{2}{|c|}{ Variables } & $\begin{array}{c}\beta \\
\text { Coefficient }\end{array}$ & $\begin{array}{c}\text { Odds Ratio } \\
(\text { OR) }\end{array}$ & P - Value & $\begin{array}{c}\text { Confidence } \\
\text { Interval (CI) }\end{array}$ \\
\hline \multirow{2}{*}{ Skeletal } & $\begin{array}{l}\text { Nasion perpendicular to } \\
\text { Pogonion (mm) }\end{array}$ & -0.06 & 0.94 & $0.020^{*}$ & $0.89-0.99$ \\
\hline \multirow{3}{*}{ Dental } & UI-SN (degrees) & 0.12 & 1.12 & $<0.001^{* *}$ & $1.07-1.18$ \\
\cline { 2 - 6 } & IMPA (degrees) & 0.11 & 1.12 & $<0.001^{* *}$ & $1.07-1.18$ \\
\cline { 2 - 6 } & Mandibular TSALD (mm) & -0.18 & 0.83 & $<0.001^{* *}$ & $0.76-0.90$ \\
\hline Soft Tissue & S-plane to Upper lip (mm) & 0.47 & 1.60 & $0.004^{*}$ & $1.16-2.20$ \\
\hline
\end{tabular}

$\mathrm{N}=240$, Binary logistic regression analysis, *p-value $\leq 0.05$, $* *$ p-value $<0.001$

dental variables showed a significant difference between the treatment groups. In an end-on class II malocclusion, the odds of extraction treatment were 1.12 times that of non-extraction treatment for every one degree upsurge in upper and lower incisor inclinations, respectively. Similarly, chances of extraction treatment were 1.6 times higher than non-extraction treatment for every $1 \mathrm{~mm}$ increase in the distance of upper lip to S-plane. The regression model states that a Class II malocclusion with half-unit molar relationship having increased upper and lower incisor inclinations and procumbent upper lip has a greater chance of being efficiently treated by extraction mechanics. 


\section{DISCUSSION}

Class II malocclusion is one of the most prevailing malocclusions in our society. ${ }^{15,16}$ The orthodontists are interested in gaining knowledge about selective parameters that could help in efficient and accurate treatment planning. Taking into consideration the soft tissue, dental cast and cephalometric values, an objective and quantifiable treatment plan could be generated for a specific individual. A full unit Class II molar relationship is usually treated with extraction, myo-functional therapy or, if the skeletal malocclusion warrants, orthognathic surgery. However, in suitable cases full unit Class II molar relationship can be corrected by non-extraction treatment strategies like molar distalization or bite jumper appliances, but these techniques are not routinely implemented.$^{1}$ On the other hand, end-on or half unit Class II molar relationship is frequently treated by both extraction and non-extraction strategies and it is not uncommon among clinicians to have variable opinion on extraction decision in such cases.

According to the statistical results of this study, the upper and lower incisor proclination and a procumbent upper lip favors an extraction decision. An odds ratio of greater than 1 in the study results is suggestive of extraction plan. An end-on class II malocclusion presents with either a normal or prognathic maxilla or a deficient mandible. In some scenarios the maxillary incisors are proclined creating increased upper lip prominence with acuteness of nasolabial angle. ${ }^{1}$ Instead, in mandibular deficient cases natural compensations are present in the form of proclined lower incisors. ${ }^{1}$ Hence treatment planning of a Class II division 1 case essentially require a four unit extraction to correct the facial esthetics, lip prominence and improve stability of occlusion with moving the incisors within the normal range. Few studies have focused on the factors that help towards the decision making process of a Class II malocclusion. The results of Paquette and co authors ${ }^{17}$ also indicate that convex facial profiles and proclined upper and lower incisors are major contributors towards an extraction decision. Similarly Bishara and associates ${ }^{13}$ stated that lip prominence, crowded arches and a Class II molar relationship is suggestive of an extraction decision. On the contrary, Guo et $\mathrm{al}^{18}$ reported that the most important factors guiding towards an extraction decision in Class II cases are increased overjet, vertical growth pattern and crowded arches. Likewise, according to Shearn and Woods ${ }^{19}$, incisor overjet is one of the major factor influencing the extraction decision. Analogous to the results of our study, Ali et $\mathrm{al}^{20}$, and Konstantonis and co-workers ${ }^{21}$ reported that incisor proclination, lip prominence and crowded arches are also one of the deciding factors in treatment of a Class I malocclusion.
The results of the current study based on end-on Class II molar relationship is dissimilar to the results of previous studies in a few aspects. ${ }^{17-20}$ Firstly, the overjet was not found to play a significant role in the extraction decision of end-on Class II molar relationship patients. Secondly, crowding which has been shown to play a key role in Class I and full unit Class II malocclusion cases, was found to play no significant role in extraction decision in cases with end-on Class II molar relationship. These results suggest that less severe Class II malocclusion, with increased overjet or mild to moderate crowding or both could be efficiently managed with a non-extraction therapeutic approach. In such cases, the clinicians could use different non-extraction modalities for correcting the crowding and molar relationship.

It is worth mentioning that the clinicians experience, biomechanical strategies and esthetic sense also has an impact on the treatment planning process, hence, the variation in results are found among different studies as these subjective variables are difficult to quantify.

\section{LIMITATIONS AND RECOMMENDATIONS}

The study, although, included a fairly large sample but the sample was collected from a single tertiary care center undermining the generalizability of the results. Besides, the norms of diagnostic parameters and morphological characteristics have certain ethnic variations which could affect the treatment decision. The authors recommend recruiting the sample from multiple institutes to give more predictable and generalizable results in future studies.

\section{CONCLUSIONS}

The current study concludes that upper and lower incisors inclinations and upper lip position are the critical factors affecting the extraction decision in adult patients with end-on Class II molar relationships. Overjet, dental crowding and the vertical growth pattern formerly reported to be other key factors in extraction decision of orthodontic patients were found to play no significant role in extraction decisionof patients with end-on Class II molar relationship.

\section{CONFLICT OF INTEREST}

None to declare

\section{REFERENCES}

1. Graber TM, Vanarsdall RL, Vig KL, editors. Orthodontics: current principles and techniques. Mosby, St. Louis; 2005. 
Ali B/ Jeelani W/ Shaikh A/

Quadeer TA/ Khan JA

2. Jasper JJ, McNamara JA Jr. The correction of interarch malocclusions using a fixed force module. Am J Orthod Dentofacial Orthop 1995;108:641-50.

https://doi.org/10.1016/S0889-5406(95)70010-2

3. Draker HL. Handicapping labio-lingual deviations: a proposed index for public health purposes. Am J Orthod 1960;46:295-305. https://doi.org/10.1016/0002-9416(60)90197-4

4. Fortini A, Lupoli M, Giuntoli F, Franchi L. Dentoskeletal effects induced by rapid molar distalization with the first class appliance. Am J Orthod Dentofacial Orthop 2004;125:697-704.

https://doi.org/10.1016/j.ajodo.2003.06.006

5. Boyd RL. Mucogingival considerations and their relationship to orthodontics. J Periodontol 1978;49:67-76.

https://doi.org/10.1902/jop.1978.49.2.67

6. Shapiro PA. Mandibular dental arch form and dimension. Treatment and post retention changes. Am J Orthod 1974;66:58-70.

7. Gallerano RL. Mandibular anterior crowding: a post retention s tudy. [Thesis.] Seattle: University of Washington, 1976.

8. Howes A. Expansion as a treatment procedure: Where does it stand today? Am J Orthod 1960;46:515-34.

9. Wholley CJ, Woods MG. The effects of commonly prescribed premolar extraction sequences on the curvature of the upper and lower lips. Angle Orthod 2003;73:386-95.

10. Katsaros C, Ripplinger B, Hogel A, Berg R. The influence of extraction versus non-extraction orthodontic treatment on the soft tissue profile. J Orofac Orthop 1996; 57:354-65.

https://doi.org/10.1007/BF02215673

11. Janson G, Mendes LM, Junqueira CH, Garib DG. Soft-tissue changes in Class II malocclusion patients treated with extractions: a systematic review. Eur J Orthod. 2016;38:631-37.

https://doi.org/10.1093/ejo/cjv083

12. Vaden JL, Williams RA, Go forth RL. Class II correction: Extraction or non extraction? Am J Orthod Dentofacial Orthop. 2018;154:860-76.

https://doi.org/10.1016/j.ajodo.2018.06.010
Identification of orthodontic extraction predictors in end-on class II malocclusion

13. Bishara SE, Cummins DM, Jakobsen JR. The morphologic basis for the extraction decision in Class II division 1 malocclusions: a comparative study. Am J Orthod Dentofac Orthop 1995;107:129-35. https://doi.org/10.1016/S0889-5406(95)70127-3

14. El Askary, Abd El Salam, and Abd El Salam El Askary. 2007. Fundamentals of esthetic implant dentistry. Ames, Iowa: Blackwell Munksgaard.

15. Sakrani H, Hussain SS, Ansari O, Hanif M. Prevalence of malocclusion in patients reporting in an orthodontic OPD of a tertiary care hospital. Pak O Dent J. 2010;2:8-13.

16. Khan S. Prevalence of malocclusion and its relation with crowding and spacing. Pak O Dent J. 2014;3:472-476.

17. Paquette DE, Beattie JR, Johnston LE Jr. A long term comparison of non extraction and premolar extraction edgewise therapy in "borderline" Class II patients. Am J Orthod Dentofacial Orthop 1992;102:1-14.

https://doi.org/10.1016/0889-5406(92)70009-Y

18. Guo Y, Han X, Xu H, Ai D, Zeng H, Bai D. Morphological characteristics influencing the orthodontic extraction strategies for Angle's class II division 1 malocclusions. Prog Orthod 2014;15:44. https://doi.org/10.1186/s40510-014-0044-y

19. Shearn BN, Woods MG. An occlusal and cephalometric analysis of lower first and second premolar extraction effects. Am J Orthod Dentofacial Orthop 2000;117:351-61.

https://doi.org/10.1016/S0889-5406(00)70240-X

20. Ali B, Shaikh A, Fida M. Factors affecting treatment decisions of Class I malocclusions. Am J Orthod Dentofacial Orthop 2018;154:234-7.

https://doi.org/10.1016/j.ajodo.2017.11.035

21. Konstantonis D, Anthopoulou C, Makou M. Extraction decision and identification of treatment predictors in Class I malocclusions. Prog Orthod 2013;19:14-47.

https://doi.org/10.1186/2196-1042-14-47 